\title{
The US COVID-19 Trends and Impact Survey: Continuous real-time measurement of COVID-19 symptoms, risks, protective behaviors, testing, and vaccination
}

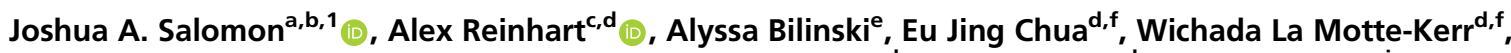 \\ Minttu M. Rönn ${ }^{g}$, Marissa B. Reitsma ${ }^{a}$, Katherine A. Morris ${ }^{h}$, Sarah LaRoccah ${ }^{h}$ Tamer H. Farag ${ }^{i}\left(\mathbb{D}\right.$, Frauke Kreuter $^{j, k}$, \\ Roni Rosenfeld ${ }^{d, f}{ }_{(D)}$ and Ryan J. Tibshirani ${ }^{c, d, f}$ \\ ${ }^{a}$ Department of Health Policy, Stanford University, Stanford, CA 94305; ${ }^{b}$ Freeman Spogli Institute for International Studies, Stanford University, Stanford, CA \\ 94305; ' Department of Statistics and Data Science, Carnegie Mellon University, Pittsburgh, PA 15213; 'Delphi Group, Carnegie Mellon University, Pittsburgh, \\ PA 15213; ' Department of Health Services, Policy and Practice, Brown School of Public Health, Providence, RI 02903; ${ }^{f}$ Machine Learning Department, Carnegie

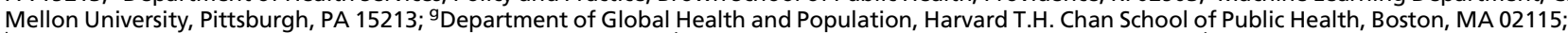 \\ ${ }^{h}$ Demography and Survey Science, Meta, Menlo Park, CA 94025; 'Health Partnerships, Meta, Menlo Park, CA 94025; 'joint Program in Survey Methodology, \\ University of Maryland, College Park, MD 20742; and ${ }^{\mathrm{k}}$ Department of Statistics, Ludwig-Maximilians-Universität Munich, Munich 80539, Germany
}

Edited by Larry Wasserman, Carnegie Mellon University, Pittsburgh, PA, and approved October 29, 2021 (received for review June 22, 2021)

The US COVID-19 Trends and Impact Survey (CTIS) is a large, crosssectional, internet-based survey that has operated continuously since April 6, 2020. By inviting a random sample of Facebook active users each day, CTIS collects information about COVID-19 symptoms, risks, mitigating behaviors, mental health, testing, vaccination, and other key priorities. The large scale of the survey-over 20 million responses in its first year of operation-allows tracking of trends over short timescales and allows comparisons at fine demographic and geographic detail. The survey has been repeatedly revised to respond to emerging public health priorities. In this paper, we describe the survey methods and content and give examples of CTIS results that illuminate key patterns and trends and help answer high-priority policy questions relevant to the COVID-19 epidemic and response. These results demonstrate how large online surveys can provide continuous, real-time indicators of important outcomes that are not subject to public health reporting delays and backlogs. The CTIS offers high value as a supplement to official reporting data by supplying essential information about behaviors, attitudes toward policy and preventive measures, economic impacts, and other topics not reported in public health surveillance systems.

COVID-19 | SARS-CoV2 | survey

D uring 2020, the coronavirus disease 2019 (COVID-19) pandemic precipitated the need for new public health surveillance to inform urgent policy decisions. Effective pandemic policy-making requires information on a broad array of indicators, including local morbidity and mortality, preventive behaviors, healthcare capacity, and economic impacts. Given the critical importance of COVID-19 trends for policy, health departments set up routine public reporting systems for tracking cases, deaths, testing, and hospitalizations (1). However, supplemental data can both augment official reporting, for example by providing additional indicators of COVID-19 prevalence not subject to reporting delays and backlogs, and supply complementary information about public behavior, attitudes toward policy and preventive measures, mental health, economic impacts, and other items not observed in public health surveillance systems.

A number of efforts have used surveys to provide supplemental surveillance data. For example, symptom-tracking smartphone apps invite users to self-report symptoms, in some cases encouraging repeated participation to enable longitudinal tracking (2-4). Other surveys have addressed broader impacts of the pandemic, such as economic consequences (5). In this paper, we present findings from the Delphi Group at Carnegie Mellon University (CMU) US COVID-19 Trends and Impact Survey (CTIS), in partnership with Facebook, which has operated continuously since April 6, 2020 and collected over 20 million responses. [An international version of the survey is described in a companion paper in this theme issue (6).]

As the largest public health survey conducted in the United States to date, CTIS is designed to facilitate detailed demographic and geographic analyses, to track trends over time, and to accommodate rapid response to emerging priorities (7). A random sample of Facebook active users are invited each day to complete a questionnaire comprising survey items on symptoms, COVID testing, social distancing, vaccination, schooling, mental health, and economic security. The survey instrument has been updated frequently to incorporate new policy-relevant topics. Results are aggregated and made publicly available, and

\section{Significance}

The US COVID-19 Trends and Impact Survey (CTIS) has operated continuously since April 6, 2020, collecting over 20 million responses. As the largest public health survey conducted in the United States to date, CTIS was designed to facilitate detailed demographic and geographic analyses, track trends over time, and accommodate rapid revision to address emerging priorities. Using examples of CTIS results illuminating trends in symptoms, risks, mitigating behaviors, testing, and vaccination in relation to evolving high-priority policy questions over 12 mo of the pandemic, we illustrate the value of online surveys for tracking patterns and trends in COVID outcomes as an adjunct to official reporting, and showcase unique insights that would not be visible through traditional public health reporting.

Author contributions: J.A.S., A.R., A.B., K.A.M., S.L., T.H.F., F.K., R.R., and R.J.T. designed research; J.A.S., A.R., A.B., E.J.C., W.L.M.-K., M.M.R., M.B.R., R.R., and R.J.T. performed research; J.A.S., A.R., A.B., E.J.C., M.M.R., and M.B.R. analyzed data; J.A.S. and A.R. wrote the paper; and A.B., E.J.C., W.L.M.-K., M.M.R., M.B.R., K.A.M., S.L., T.H.F., F.K., R.R., and R.J.T. revised the paper

Competing interest statement: This material is based on work supported by gifts from Facebook, Google.org, and grants from the Centers for Disease Control and Prevention.

This article is a PNAS Direct Submission.

This open access article is distributed under Creative Commons Attribution License 4.0 (CC BY).

${ }^{1}$ To whom correspondence may be addressed. Email: salomon1@stanford.edu.

Published December 13, 2021 
microdata are available under institutional data use agreement, in both cases with fewer than $3 \mathrm{~d}$ of lag. These data provide information at a level of geographic and temporal detail that can supply essential inputs into short-term decision-making and longer-term strategic planning. These data also facilitate retrospective analysis of patterns, trends, and associations, supporting longer-term research on health policy decisions and the impacts of the pandemic.

In this study, we first compare COVID-19 indicators from CTIS with publicly reported case, hospitalization, and mortality data between April 2020 and April 2021. Despite potential limitations of our internet-based sample and the voluntary nature of the survey, we demonstrate high correspondence between the two, with CTIS less affected by holiday-related reporting anomalies. Second, we examine patterns and trends in symptoms, risks, mitigating behaviors, testing, and vaccination in US states and localities, in relation to evolving high-priority policy questions over 12 mo of the pandemic. The findings illustrate the value of online surveys for tracking patterns and trends in COVID-related outcomes as an adjunct to official reporting, while also showcasing insights that are only possible through a large-scale survey effort.

\section{Methods}

Sampling and Recruitment. The US CTIS launched on April 6, 2020 and has run continuously since that time, with an average of more than 350,000 people participating each week over the first year of operation. The survey is implemented by the Delphi Group at CMU, with participants recruited via the Facebook platform. Every day, Facebook invites a new sample of active users ages $18 \mathrm{y}$ or older to participate in the survey. Facebook uses stratified random sampling within US states to randomly select a sample of its users to see the survey invitation at the top of their News Feed. Users who click on the invitation are taken to the CMU-administered survey hosted on Qualtrics. To ensure privacy, Facebook does not see any individual survey response during or after the data collection. The survey is available in English, Spanish, Brazilian Portuguese, Vietnamese, French, and simplified Chinese.

Survey Design. The survey instrument was deployed in multiple waves from launch through April 5, 2021, with contents of each survey version summarized in Table 1. Revisions are ongoing as new public health needs arise. A number of core items have been included consistently across all survey versions, including questions about symptoms, contacts, and demographics. Key additions include items on mask wearing and occupation, added in September 2020, seasonal flu vaccination and schooling, added in November 2020, and COVID-19 vaccination, added in December 2020. As of April 5, 2021, the range of survey items spanned the following broad categories: household and individual symptoms, common comorbidities, contact patterns and mitigating behaviors, testing and diagnosis, worry and financial impact, schooling, vaccination, and demographics.

Full versions of all survey instruments can be found at https://cmu-delphi. github.io/delphi-epidata/symptom-survey/coding.html.

Weighting. Analytic weights have been developed to adjust for differences between Facebook users and the United States population, and to adjust for biases related to coverage and nonresponse (8). When Facebook links users to the survey, it generates a random unique identifier that is passed to CMU. For users who complete the survey, CMU returns the corresponding identifiers to Facebook, which then calculates analytic weights in two steps:

1. To adjust for nonresponse bias, Facebook calculates the inverse probability that sampled users complete the survey using their age, gender, and geographical variables, as reported on their Facebook profiles, as well as other characteristics known to correlate with nonresponse. The inverse probabilities are then used to create weights for responses, after which the survey sample reflects the active adult user population on Facebook.

2. To adjust for coverage bias, Facebook poststratifies the weights created in the first step so that the distribution of age, gender, and state or territory of residence in the survey sample reflects that of the general population.

The analytic weight does not identify the survey respondent. The weight for an individual is scaled to approximate the number of people in the adult population represented by that individual based on age, gender, location, and date. Facebook passes these weights to CMU. To protect respondent privacy, $\mathrm{CMU}$ cannot use these weights to identify specific Facebook users, and Facebook never receives individual survey responses and cannot link them to specific users.

Analysis. In this study we examined a range of different outcomes measured in the CTIS over the period April 6, 2020 to April 5, 2021. We analyzed both aggregated macrolevel data and individual-level data to highlight some of the key questions that may be examined using CTIS. Across the examples presented in this paper, we have stratified analyses by individual characteristics, including age, race/ethnicity (using categories consistent with National Center for Health Statistics), and occupation, and by geographic divisions, including Census region, Census division, state, and county.

Table 1. Summary of survey waves deployed between April 6, 2020 and April 5, 2021

\begin{tabular}{|c|c|c|c|}
\hline Wave* & Contents & Start date & $n$ (in millions) \\
\hline 1 & $\begin{array}{l}\text { Household and individual symptoms } \\
\text { Common comorbidities } \\
\text { Contacts with others } \\
\text { Anxiety, depression } \\
\text { Financial impact } \\
\text { Demographics (age, gender) }\end{array}$ & April 6, 2020 & 1.1 \\
\hline 2 & $\begin{array}{l}\text { New question: Symptoms among "people in your local community that you know personally" } \\
\text { Minor textual revisions }\end{array}$ & April 15, 2020 & 2.6 \\
\hline 3 & $\begin{array}{l}\text { Translated into simplified Chinese, Spanish, French, Brazilian Portuguese, Vietnamese } \\
\text { Minor textual revisions }\end{array}$ & May 21, 2020 & 7.4 \\
\hline 4 & $\begin{array}{l}\text { New questions: Medical care sought, COVID testing and results, mask wearing, social isolation } \\
\text { Additional demographics, including race, ethnicity, occupation, education } \\
\text { Textual revisions } \\
\text { Some unused items removed }\end{array}$ & September 8, 2020 & 3.0 \\
\hline 5 & $\begin{array}{l}\text { New questions: Seasonal flu vaccination, schooling, and school precautions } \\
\text { Textual revisions }\end{array}$ & November 24, 2020 & 1.3 \\
\hline 6 & New questions: Vaccine intent. Vaccine status item enabled on January 6, 2021 & December 19, 2020 & 1.2 \\
\hline 7 & Textual revisions to vaccine intent items & January 12, 2021 & 1.3 \\
\hline 8 & $\begin{array}{l}\text { New questions: Reasons for vaccine hesitancy, vaccine dosing } \\
\text { Minor textual revisions }\end{array}$ & February 8, 2021 & 0.9 \\
\hline 10 & $\begin{array}{l}\text { New questions: Appointments for COVID vaccines, information about getting vaccinated } \\
\text { Textual revisions }\end{array}$ & March 2, 2021 & 1.4 \\
\hline
\end{tabular}

*There was no Wave 9 survey. The numbering of waves skipped from 8 to 10 to synchronize numbering conventions with the international version of the survey. 
To examine the representativeness of the study sample, we compared characteristics of the sample to data from the American Community Survey 2019 (ACS) supplemental estimates.

We evaluated reported symptoms and symptom patterns in comparison to surveillance data on confirmed COVID-19 hospitalizations from the Department of Health and Human Services (9) and reported COVID-19 cases and mortality aggregated by the Johns Hopkins University Center for Systems Science and Engineering (10). To summarize relevant symptom patterns, we defined "COVID-like illness" (CLI) as reporting a fever of at least $100^{\circ} \mathrm{F}$, along with cough, shortness of breath, or difficulty breathing, in line with a working definition of $\mathrm{CLI}$ used for syndromic surveillance purposes beginning in early 2020 . A second indicator, which we call "CLI in Community," was based on responses to an item on the survey that asks whether respondents know someone personally in their community who is ill with COVID-like symptoms. We also compared reported diagnoses in CTIS to cumulative diagnoses in surveillance data.

To illustrate the utility of individual-level data to provide detailed information on characteristics that may be relevant to transmission risk, we compared reported diagnoses and reports of working outside of the home while symptomatic across occupational categories. To evaluate time trends in risk exposures and mitigating behaviors, we examined reported contacts, mask use, and use of public transit over time, across a range of stratifying variables, including counties grouped by levels on the Centers for Disease Control and Prevention (CDC) Social Vulnerability Index (SVI) (11), which is a composite measure constructed based on 15 social variables measured at the census tract level, age groups, and Census regions.
Finally, as an illustration of the information value of the survey at various levels of granularity, we assessed reported COVID-19 vaccination intent stratified by individual characteristics and across counties.

The study was approved by the CMU Institutional Review Board, under protocol STUDY2020_00000162. All respondents gave informed consent before participating in the survey.

\section{Results}

Characteristics of the Study Sample. As of April 5, 2021, a total of 20.2 million responses had been collected in the US CTIS. Table 2 summarizes characteristics of the survey respondents. Compared to the weighted sample, the unweighted sample had a higher proportion of women (66\% vs. $52 \%)$ and a slightly higher proportion of respondents between ages 25 and $64 \mathrm{y}$ (72\% vs. $68 \%)$. Household size and prevalence of at least one comorbidity were similar in the unweighted and weighted samples.

Compared to 2019 ACS supplemental estimates, the weighted survey sample slightly overrepresented women, but had a broadly comparable age distribution and matched the ACS distribution across geographic regions. The weighted sample included a larger proportion of respondents with greater than a high school education, and a much smaller proportion

Table 2. Characteristics of the study sample, compared to 2019 ACS supplemental estimates

\begin{tabular}{|c|c|c|c|c|}
\hline & Number & Unweighted proportion (\%) & Weighted proportion (\%) & Census proportion (\%) \\
\hline All responses & $20,249,152$ & & & \\
\hline \multicolumn{5}{|l|}{ Gender } \\
\hline Female & $11,409,227$ & 66.3 & 52.3 & 50.8 \\
\hline Male & $5,613,674$ & 32.6 & 46.2 & 49.2 \\
\hline Nonbinary/self-described & 174,124 & 1.0 & 1.5 & - \\
\hline \multicolumn{5}{|l|}{ Age groups } \\
\hline $18-24$ & $1,001,345$ & 5.8 & 10.7 & 11.9 \\
\hline $25-34$ & $2,856,685$ & 16.5 & 16.4 & 17.9 \\
\hline $35-44$ & $3,212,187$ & 18.5 & 16.5 & 16.4 \\
\hline $45-54$ & $3,129,334$ & 18.1 & 17.7 & 16.0 \\
\hline $55-64$ & $3,337,427$ & 19.3 & 17.5 & 16.6 \\
\hline $65-74$ & $2,752,379$ & 15.9 & 15.3 & $21.2^{*}$ \\
\hline$\geq 75$ & $1,035,551$ & 6.0 & 6.0 & \\
\hline \multicolumn{5}{|l|}{ Education $^{\dagger}$} \\
\hline Less than high school & 226,284 & 3.1 & 4.0 & 12.0 \\
\hline High school or equivalent & $1,152,727$ & 16.0 & 17.2 & 27.1 \\
\hline Some college, no degree & $1,744,155$ & 24.1 & 24.5 & 20.4 \\
\hline Associate's degree & 829,618 & 11.5 & 11.2 & 8.5 \\
\hline Bachelor's degree & $1,765,207$ & 24.4 & 23.6 & 19.7 \\
\hline Graduate or professional degree & $1,505,785$ & 20.8 & 19.5 & 12.3 \\
\hline \multicolumn{5}{|l|}{ Region } \\
\hline Northeast & $3,426,497$ & 17.4 & 17.6 & 17.4 \\
\hline Midwest & $4,791,585$ & 24.3 & 20.9 & 20.8 \\
\hline South & $7,328,178$ & 37.2 & 37.9 & 38.0 \\
\hline West & $4,160,577$ & 21.1 & 23.6 & 23.8 \\
\hline \multicolumn{5}{|l|}{ Household size } \\
\hline 1 & $2,698,400$ & 13.7 & 13.3 & \\
\hline 2 & $6,668,445$ & 33.9 & 31.9 & \\
\hline $3-5$ & $8,489,946$ & 43.1 & 43.7 & \\
\hline $6-10$ & $1,476,637$ & 7.5 & 8.7 & \\
\hline$>10$ & 352,267 & 1.8 & 2.4 & \\
\hline \multicolumn{5}{|l|}{ Date of completion } \\
\hline April-June 2020 & $6,814,488$ & 33.7 & 23.6 & \\
\hline July-September 2020 & $5,280,596$ & 26.1 & 25.2 & \\
\hline October-December 2020 & $3,832,698$ & 18.9 & 25.2 & \\
\hline January-March 2021 & $4,131,800$ & 20.4 & 24.7 & \\
\hline April 2021 & 189,570 & 0.9 & 1.4 & \\
\hline At least 1 comorbidity & $9,949,181$ & 53.7 & 52.0 & \\
\hline
\end{tabular}

*Value reported for 65 y and older in ACS.

${ }^{\dagger}$ Value reported for adults 25 and older in ACS; only collected in CTIS beginning in Wave 4. 


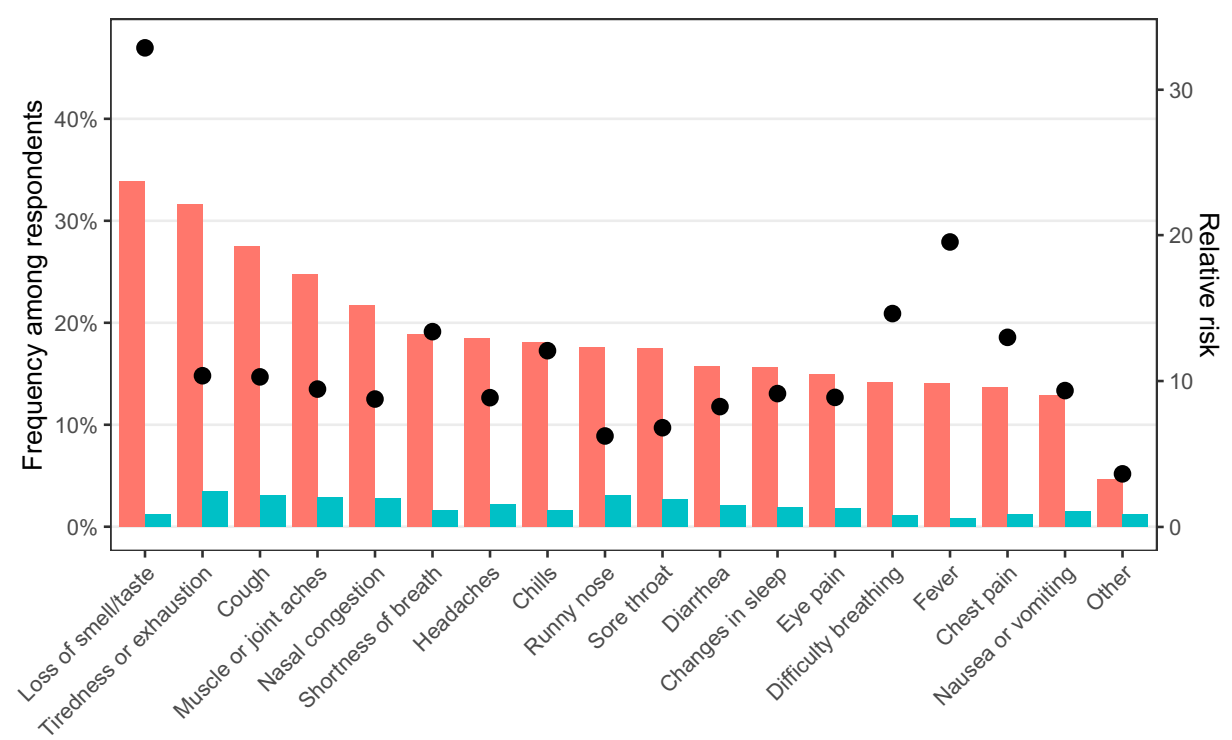

Group Tested positive $\quad$ All others

Fig. 1. Frequency of reported new or unusual symptoms, pooled over respondents to the CTIS, September 8 , 2020 to April 5, 2021. Respondents are grouped by whether they indicated they tested positive in the past $14 \mathrm{~d}$. Dots indicate the ratio of frequency among those who tested positive compared to all others.

with less than a high school education, suggesting the presence of a sampling or response bias correlated with education. This bias has remained consistent over time. As the weights provided by Facebook do not account for education, the weighting did not correct this bias.

COVID-19 Symptoms and Diagnoses. A large fraction of daily respondents reported new or unusual symptoms consistent with COVID-19 (Fig. 1). The most common single new or unusual symptom among all respondents was "tiredness or exhaustion," with a prevalence of $3.9 \%$ over Waves 4 through 10 . Patterns of symptoms were notably different among the subset of respondents who reported testing positive for COVID-19, compared to all other respondents, including a substantially higher probability of reporting loss of smell or taste (34\% compared to $1.2 \%$ ).

Fig. 2 compares time series for three indicators from the CTIS (reported anosmia, CLI, and CLI in community) against
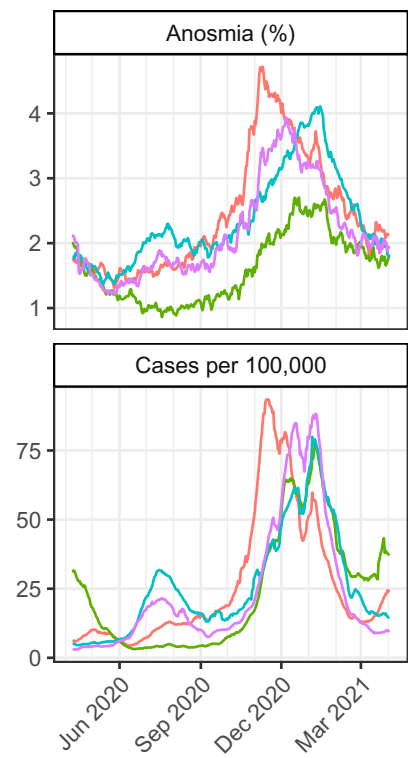
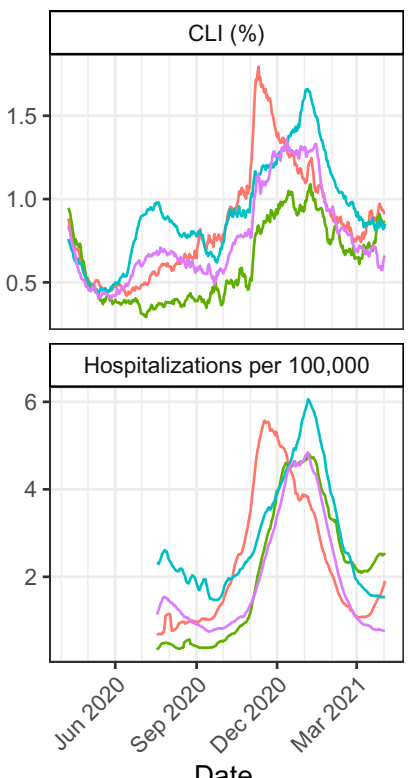
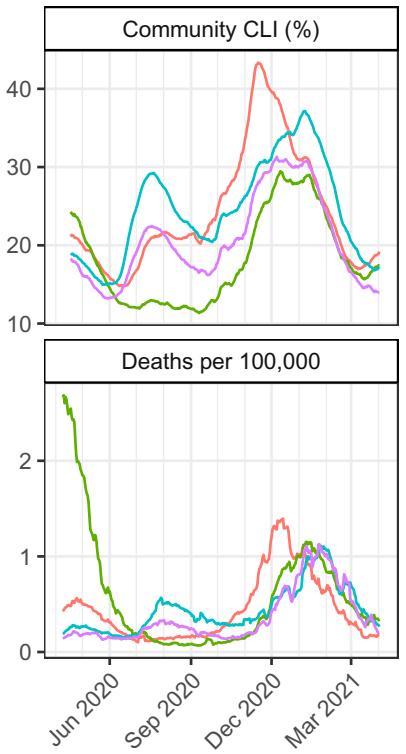

$$
\text { Census region — Midwest — Northeast — South — West }
$$

Fig. 2. Trends in anosmia, CLI, CLI in community, confirmed cases, hospitalizations, and deaths, by Census region, April 6, 2020 to April 5, 2021. 


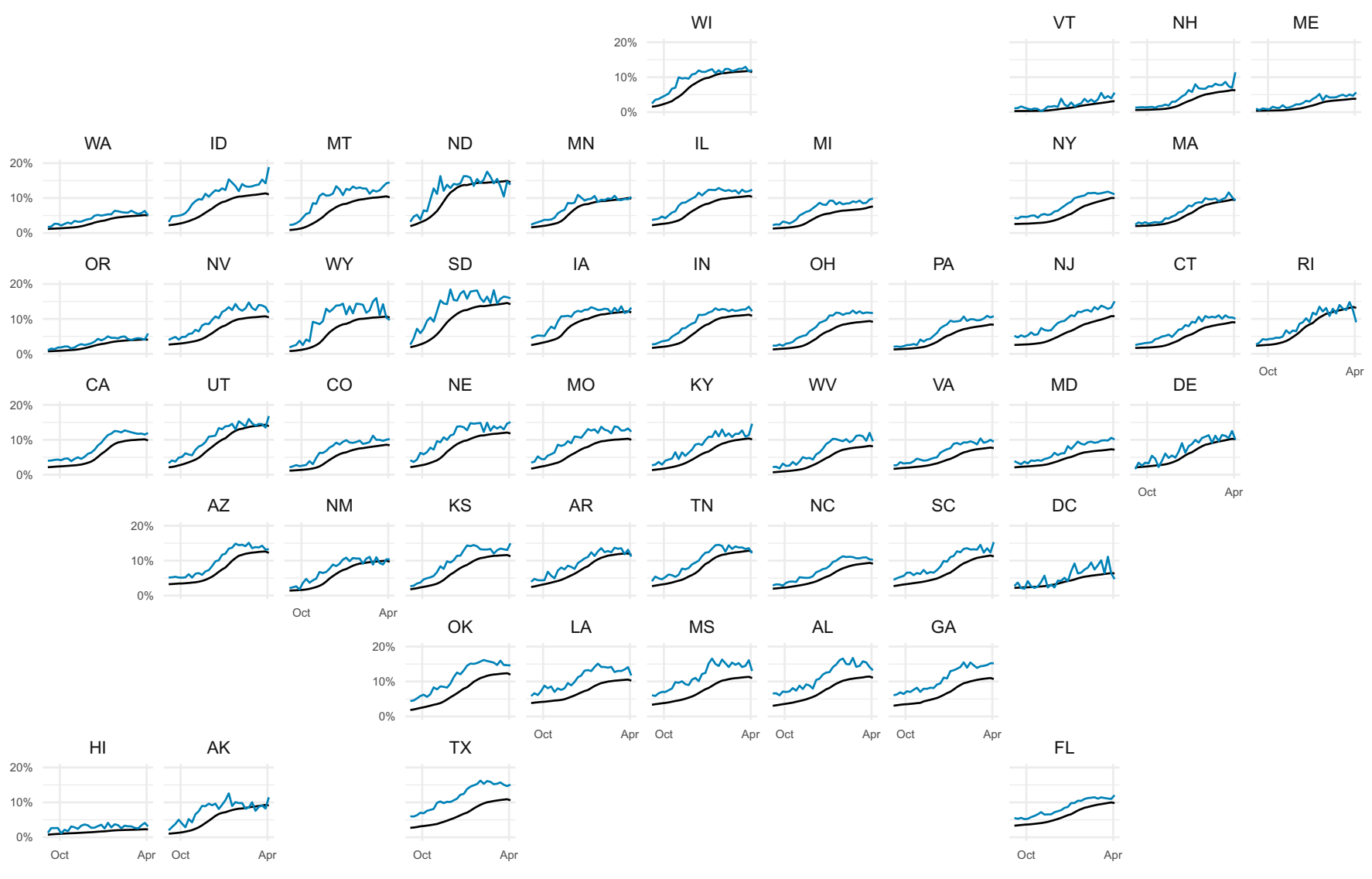

— CTIS — Surveillance

Fig. 3. Comparison of proportion of respondents in CTIS reporting ever having tested positive for COVID-19 and cumulative proportion of adult population with confirmed COVID diagnosis, by state, September 8, 2020 through April 5, 2021.

the three main surveillance indicators that have been used to monitor trends in the epidemic (confirmed cases, hospitalizations, and deaths) stratified by Census region. Over the period April 6, 2020 to April 5, 2021, the three survey indicators tracked both broad temporal trends and regional patterns in the surveillance indicators, and several notable features are evident in the comparison. First, the survey-based indicators were less susceptible to the daily fluctuations and reporting anomalies that appeared in cases and deaths, including abrupt discontinuities around certain holiday periods. Second, trends and patterns in anosmia were similar to patterns in CLI, and the anosmia series provided a closer match than the other two survey indicators to the trends and patterns observed in COVID19 hospitalizations, mirroring temporal peaks and comparative levels across regions over different waves of the epidemic. Third, CLI in community provided the most temporally stable signals while also expressing broad differences over time and space that were generally similar to those in other indicators. In a companion paper, we performed extensive correlation analyses between reported cases and various auxiliary indicators, including the survey-based CLI and CLI in community signals (12), showing strong correlations between cases and these two survey signals during much of the pandemic.

The CTIS includes questions about testing and diagnosis, which since September 8, 2020 have been asked of all respondents. Fig. 3 compares weekly CTIS estimates of the proportion of adults reporting that they have ever had a positive test for COVID-19 against cumulative diagnoses from surveillance reports by state in the same week. State surveillance reports were adjusted, using 2019 population estimates and CDC linelevel demographic data on confirmed COVID-19 cases, to produce estimated diagnosis rates among the state's population over age $18 \mathrm{y}$. As of April 5, 2021, reported diagnoses in the survey ranged from $3.1 \%$ in Hawaii to $19 \%$ in Idaho, and the correlation between survey reported diagnoses and surveillance reports at the state level was 0.83 , indicating strong convergent validity.

Transmission Risk by Individual Characteristics. Since Wave 4, the CTIS has included questions about occupation, which can offer valuable insights into exposures among essential workers and also supply signals of where transmission may be concentrated. Using responses from January 2021, we examined the probability of reporting a positive COVID-19 test across different occupation categories, as well as the probability of reporting working outside the home while having symptoms consistent with COVID-19 (Table 3). Substantial heterogeneity appeared across broad groups of occupation. The large proportion of people reporting never having been tested indicates the limitations of passive surveillance. Combining questions on symptoms, testing, and working into a single indicator, we examined the fraction of people who reported both working outside the home and currently having atypical symptoms; results ranged from more than $15 \%$ for respondents in food preparation and serving related occupations, to $4 \%$ of those in arts, design, entertainment, sports, and media. 
Table 3. Reporting testing, symptoms, and working outside the home, by reported occupation category, in January 2021

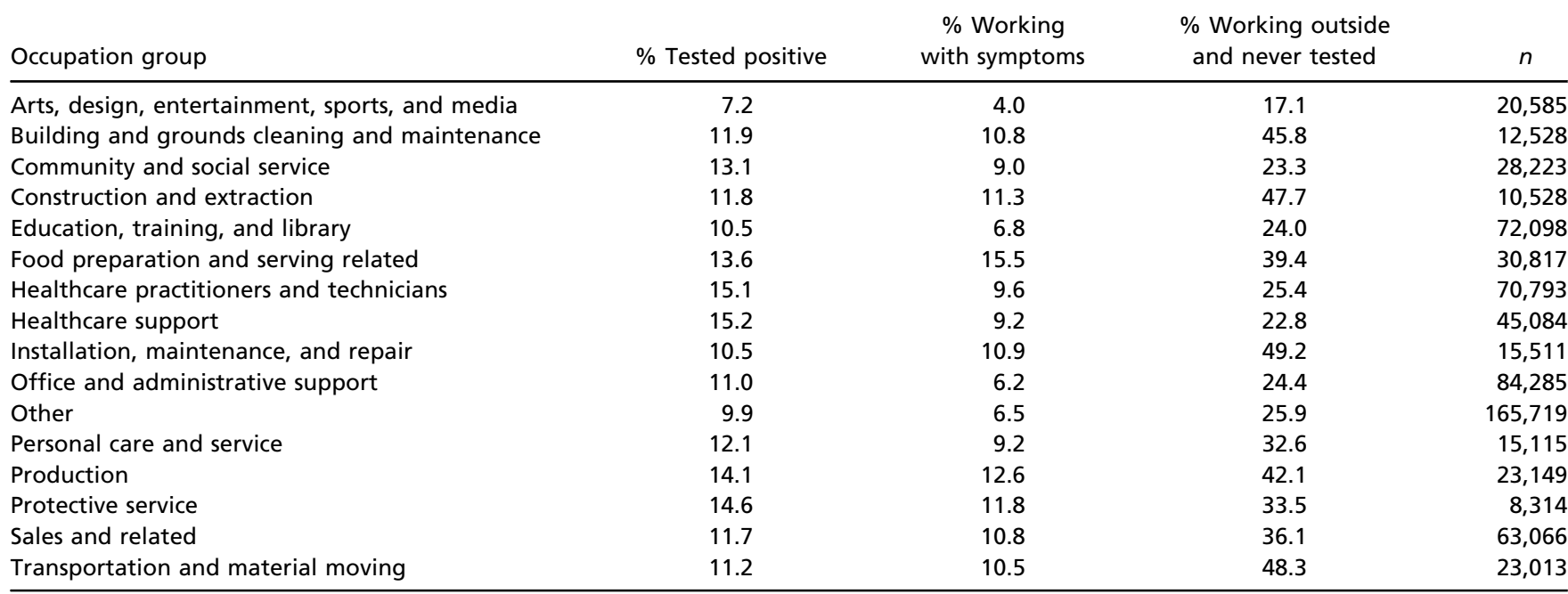

*There was no Wave 9 survey. The numbering of waves skipped from 8 to 10 to synchronize numbering conventions with the international version of the survey.

Mitigating Behaviors and Policy Analysis. A core set of questions included since the launch of the survey has addressed contacts and preventive behaviors. The survey has been amended over time to augment these items, with the addition of questions on mask use and specific high-risk behaviors in September 2020. In the context of recurrent surges in COVID-19 around the country over the course of 2020 and 2021, these items have illuminated how contacts and mitigating behaviors can shift in response to changes in local COVID-19 risk, sometimes preceding policy changes. For example, Fig. 4 shows selected variables relating to contacts and preventive behaviors over the period September 2020 to April 2021. Responses indicate sharp increases in riskreducing behaviors during November and December as cases surged-including reduced contacts, increased use of masks, and reduced use of public transit—-followed by relaxation of mitigating behaviors over the period January to April 2021 as cases fell.

Individual-level data allow for geographically detailed analysis that can also be disaggregated by demographic features. In Fig. 4,
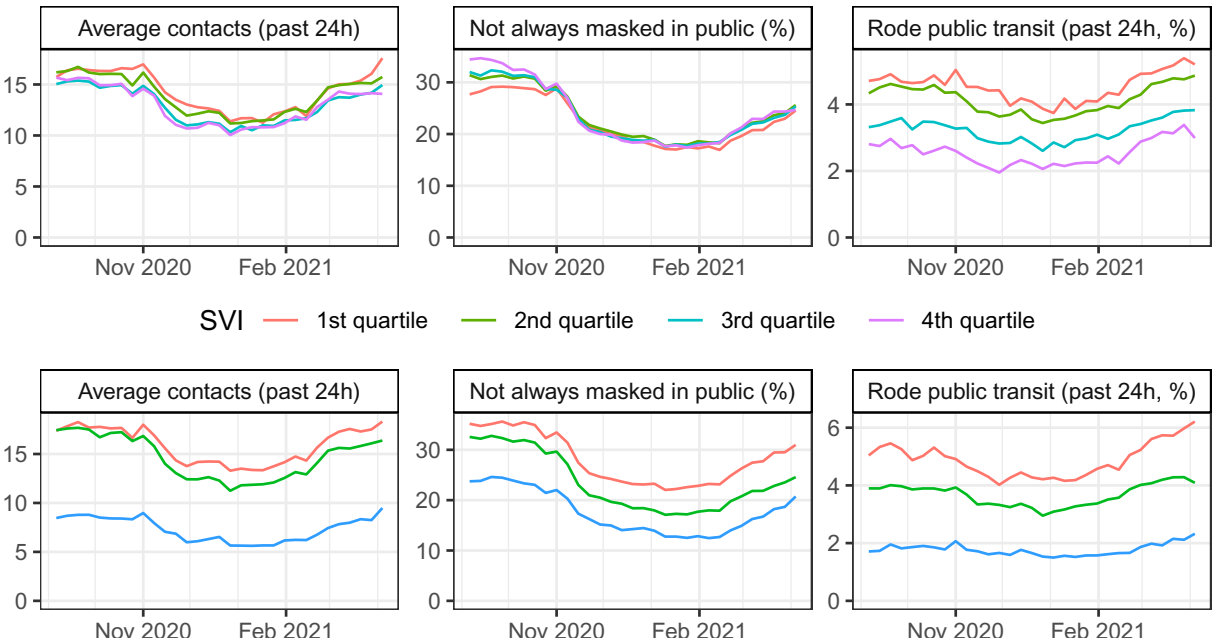

Age - 18-34-35-64-65+
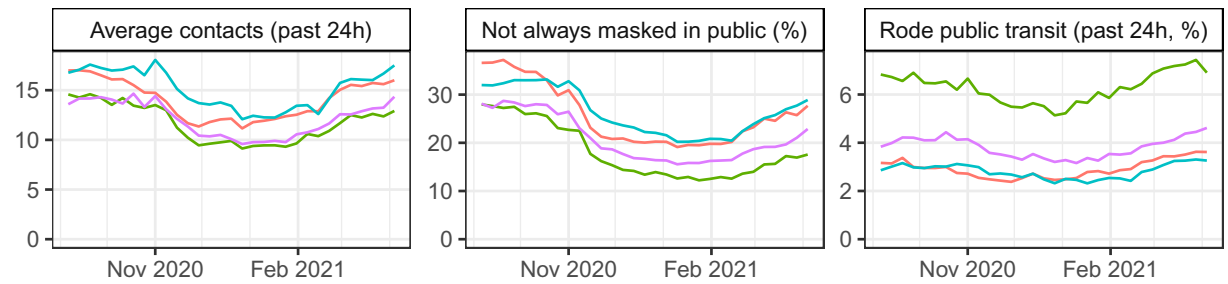

Region - Midwest - Northeast — South - West

Fig. 4. Contacts, mask use, and use of public transport, by quartile of counties grouped by the CDC SVI (Top), age group (Middle), and Census region (Bottom), September 8, 2020 to April 5, 2021 
A
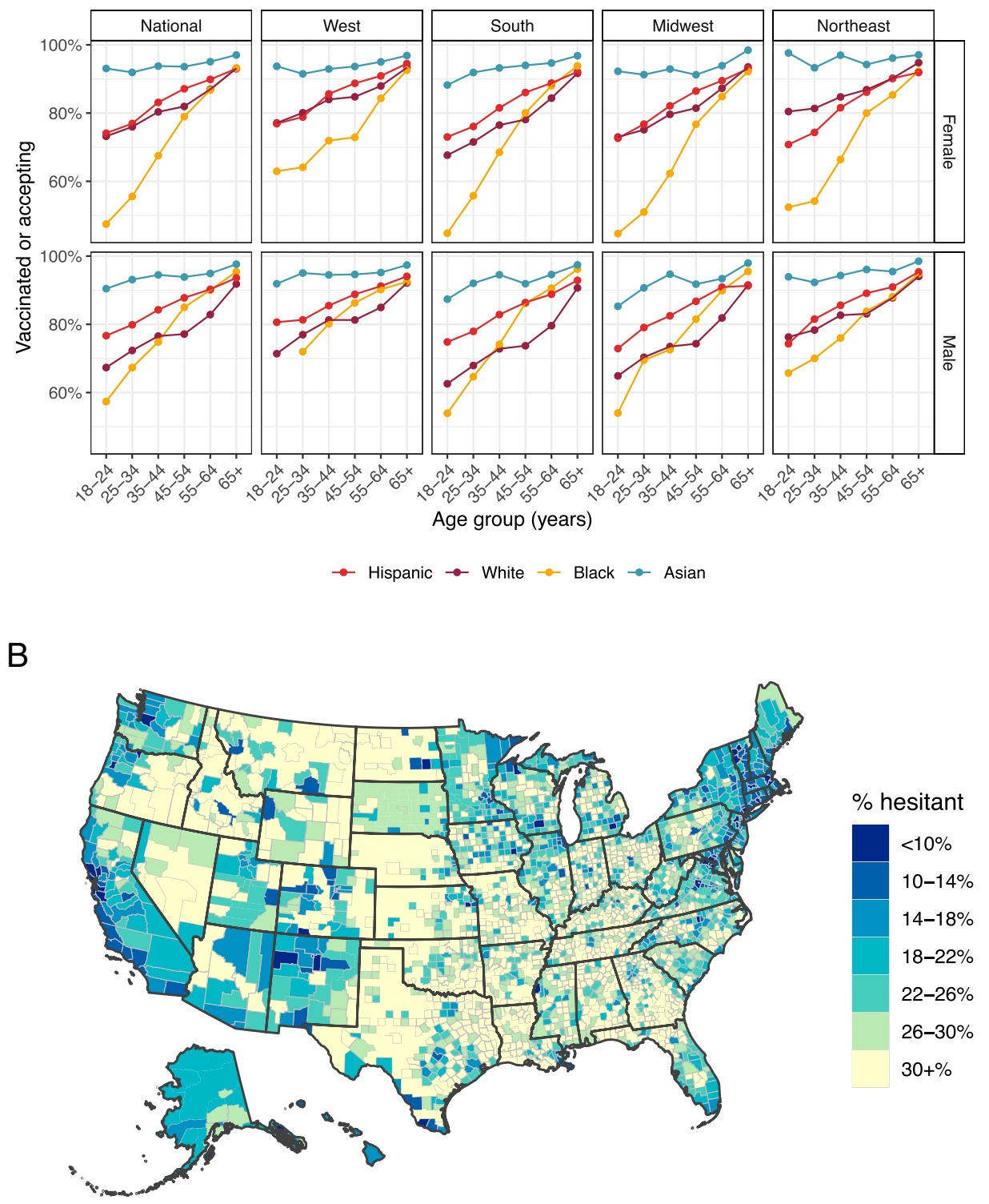

Fig. 5. Reported vaccine acceptance and hesitancy by age group, race/ethnicity, gender, and Census region $(A)$ and by county $(B)$ during March 2021. Note: Results are pooled over the period March 1, 2021 to April 5, 2021. The map displays results computed for specific counties having at least 50 responses recorded over that period, with all other counties in a state combined into a residual group.

results are stratified in three different ways to illustrate this: by quartiles of the CDC SVI, by age, and by US Census region. There were minimal differences across counties grouped by the SVI in reported mask use, moderately higher contacts among those living in more vulnerable communities, and substantially higher use of public transit in more vulnerable counties. The second row shows age differences, which indicate a pronounced gradient of higher risk mitigation among older respondents, especially with respect to reduced contacts. The third row describes regional patterns that vary across indicators, with higher contacts and lower mask use in the South and Midwest regions compared to the Northeast and West, but greater use of public transit in the Northeast and West compared to South and Midwest.

Vaccination and Vaccine Acceptance. Since December 19, 2020, the CTIS has included questions on vaccination intent, and since January 6, 2021, the survey has asked about vaccination status. The combination of geographic and demographic resolution in the survey allows a uniquely detailed view on vaccination acceptance and hesitancy across different United States population groups. Fig. $5 A$ displays results by age group, race/ ethnicity, gender, and Census region, pointing to high levels of acceptance among older respondents in all categories, but lower and more variable results at younger ages. (Respondents may identify as nonbinary or self-describe their gender, but this group was typically too small to break out and report reliable hesitancy estimates by region.) Fig. $5 B$ shows the percentage of respondents indicating that they would probably not or definitely not get vaccinated across United States counties, indicating regional patterns but also high variability across counties within a given state. As the vaccination campaign slows across the country, high-resolution information on vaccine acceptance can inform policies that aim to increase uptake toward the goal of high levels of population immunity against COVID-19. 


\section{Discussion}

As SARS-CoV-2 spread throughout the United States during 2020 and into 2021, and policy makers faced decisions that would profoundly impact all sectors of society, the breadth and depth of information needed to support these decisions vastly exceeded the availability of data collected through existing surveillance systems designed to capture reported COVID-19 cases, hospitalizations, and deaths. A number of efforts to fill the urgent need for additional information relied on novel data collection and dissemination platforms that leveraged mobile phone technology and new media. In this study, we describe one of these efforts, the CTIS, which is the largest continuous health survey ever conducted in the United States, in operation since April 6, 2020, with more than 20 million responses collected over the first year of operation.

Comparisons to routine sources of surveillance information point to both the face validity and incremental value of the CTIS. Time trends and geographic patterns in COVID-19 outcomes measured in the CTIS-including specific symptoms strongly associated with SARS-CoV-2 infection, such as anosmia, syndromic patterns, such as COVID-like-illness, and the novel CTIS measure of CLI in community-mirror broad temporal and spatial features in standard surveillance measures on confirmed COVID-19 cases, hospitalizations, and deaths, while in many cases avoiding data artifacts and reporting anomalies that affect the official measures.

In this study we have highlighted several examples of how attributes of CTIS give it particular value and salience as an information platform for public health policy. The scope, scale, and recruitment strategy used in the survey support analysis at high geographic and temporal resolution, allowing detection of local trends on short timescales not available through other surveys, and accommodate a high level of stratification on relevant individual characteristics. Several examples illustrate the benefits of this granularity, including the ability to compare risks and preventive behaviors by occupational category, with further stratification possible by demography and geographic location; ability to describe variation in intentions; and use of key mitigating measures, including physical distancing, masking, and vaccination. Regular updating of the survey has enabled the survey content to adapt alongside the evolving policy response, for example through addition of survey items on mask use in September 2020, school mitigation strategies in November 2020, and vaccination in December 2020.

Other studies have used data from the CTIS to answer specific questions about key COVID-19 impacts and policies. A number of studies have analyzed relationships between reported risk-mitigating behaviors in the CTIS and other outcomes. For example, Rebeiro et al. (13) examined reported mask-wearing behavior as an outcome in relation to statewide mask-wearing requirements. Rader et al. (14) examined the relationship between mask wearing and physical distancing as measured in CTIS and measures of community transmission. Bilinski et al. (15) assessed trends across states in a number of indicators on risk perception and preventive behaviors in relation to COVID19 case rates. Other studies have used CTIS measures to explore correlates of variation in risk. For example, Flaxman et al. (16) computed relative infection rates for healthcare workers vs. other respondents using information from the survey on occupation, testing, and test results. Lessler et al. (17) have assessed reported risks of COVID-19-related outcomes, including CLI, anosmia, or a positive COVID-19 test, in relation to whether a household includes a child who attends in-person schooling, and reported school-based mitigation measures.

Symptom measures from the survey have also been used to aid in forecasting of COVID cases and deaths. Through the COVID-19 Forecast Hub, the CDC collects standardized forecasts from dozens of teams. Rodríguez et al. (18) incorporated symptom surveillance data from CTIS into a deeplearning framework for real-time forecasting. In a companion paper in this issue (19), we demonstrate that symptom surveillance data and other auxiliary data streams (such as medical insurance claims) can improve forecasting and hotspot prediction accuracy over short (1 to $3 \mathrm{wk}$ ) time intervals.

Several limitations are important to note. First, because the survey uses Facebook active users as its sampling frame and because participation in the survey is strictly voluntary, respondents may not be fully representative of the United States population, despite incorporation of survey weights, which adjust for nonresponse and coverage biases based on a limited number of covariates. Comparison to the ACS indicates that our sample overrepresents respondents who are college-educated. Research users of the survey microdata can use additional demographic or other survey variables to construct improved poststratification adjustments to correct this for their purposes. However, any nonresponse biases not accounted for by Facebook's nonresponse weights would be much more difficult to correct.

Additionally, many of the outcome measures related to COVID-19 are based on self-reports, which may diverge from more objective measures due to recall bias, social desirability bias, and other sources of survey bias and measurement error. On the other hand, broad comparisons of indicators, such as cumulative COVID-19 diagnoses, suggest that measurement of key COVID-19 outcomes are relatively robust to response biases that may be present in the sample. Ultimately, the value of such a large-scale survey is not in accuracy afforded by its sample size, since survey biases persist no matter the size of the survey; smaller surveys, more carefully constructed to reduce sampling biases, would likely yield more accurate estimates (20). Instead, since these survey biases are unlikely to change rapidly in time or in space, CTIS can accurately track trends in key signals, even if the daily point estimates are systematically biased. This is demonstrated by the strong correlations between survey estimates of CLI in community and reported COVID case rates, for example; while CLI in community is not an unbiased population estimate of COVID case rates, it nonetheless provides useful information about trends in cases. The principal value of CTIS is hence in the detailed spatial and demographic comparisons it makes possible, and in its ability to track changes continuously over time and correlate them with key outcome measures.

Although CTIS was initially designed with a relatively limited scope, including a particular focus on syndromic surveillance, its value has ultimately derived in large part from its flexibility as a surveillance platform that can be rapidly adapted to changing information needs. Running a survey of this size has involved many challenges, particularly as it expanded to include key measures of public knowledge, attitudes, and behaviors, and as public health needs evolved continuously during the pandemic. Despite these challenges, however, CTIS has provided both a valuable public information resource during a global health emergency, as well as a potential model for ongoing health surveillance needs. Similar online surveys are likely to play important roles in future epidemics and pandemics by supplementing public reporting systems with information that is difficult to gather any other way.

Data Availability. Survey microdata are not publicly available because survey participants only consented to public disclosure of aggregate data, and because the legal agreement with Facebook governing operation of the survey prohibits disclosure of microdata without confidentiality protections for respondents. Deidentified microdata are available to researchers under a Data Use Agreement that protects the confidentiality of respondents. Access can be requested online (https://cmu-delphi.github.io/delphi-epidata/ symptom-survey/data-access.html). Requests are reviewed by the Carnegie Mellon University Office of Sponsored Programs and Facebook Data for Good. County- and state-level aggregates of key variables are publicly available in the COVIDcast API, described in detail in a companion paper (12), and are 
presented in an interactive online dashboard (https://delphi.cmu.edu/covidcast survey-results/?date=20211103). Demographic breakdowns of key variables over time are available for public download at https://cmu-delphi.github.io/ delphi-epidata/symptom-survey/contingency-tables.html. In order to safeguard the privacy of survey respondents, aggregate data are publicly presented only for cells containing at least 100 respondents. All code used to generate figures and tables in the paper is deposited with Zenodo (https://doi.org/10.5281/ zenodo.5639567).

1. Centers for Disease Control and Prevention, COVID Data Tracker. https://covid.cdc. gov/covid-data-tracker/. Accessed 16 June 2021.

2. W. E. Allen et al., Population-scale longitudinal mapping of COVID-19 symptoms, behaviour and testing. Nat. Hum. Behav. 4, 972-982 (2020).

3. A. T. Chan, J. S. Brownstein, Putting the public back in public health-Surveying symptoms of Covid-19. N. Engl. J. Med. 383, e45 (2020).

4. C. Menni et al., Real-time tracking of self-reported symptoms to predict potential COVID-19. Nat. Med. 26, 1037-1040 (2020).

5. United States Census Bureau, Household Pulse Survey (COVID-19). https://www. census.gov/programs-surveys/household-pulse-survey.html. Accessed 16 June 2021.

6. C. M. Astley et al., Global monitoring of the impact of the COVID-19 pandemic through online surveys sampled from the Facebook user base. Proc. Natl. Acad. Sci. U.S.A. 118, e2111455118 (2021).

7. F. Kreuter et al., Partnering with a global platform to inform research and public policy making. Surv. Res. Methods 14, 159-163 (2020).

8. N. Barkay et al., Weights and methodology brief for the COVID-19 symptom survey by University of Maryland and Carnegie Mellon University, in partnership with Facebook. arXiv [Preprint] (2020). https://arxiv.org/abs/2009.14675 (Accessed 16 June 2021).

9. United States Department of Health and Human Services, COVID-19 reported patient impact and hospital capacity by state timeseries. https://healthdata.gov/dataset/ COVID-19-Reported-Patient-Impact-and-Hospital-Capa/qqte-vkut. Accessed 16 June 2021.

10. E. Dong, H. Du, L. Gardner, An interactive web-based dashboard to track COVID-19 in

real time. Lancet Infect. Dis. 20, 533-534 (2020).
ACKNOWLEDGMENTS. We thank Taylor Arnold, Logan Brooks, Nat DeFries, and Kathryn Mazaitis from Delphi for contributions to survey data processing systems; Adrianne Bradford, Don Burke, Samantha Chiu, Curtiss Cobb, Xiaoyi Deng, Evan MacKay, Jonathan McKay, Stephanie Perniciaro, Stanley Presser, Liz Stuart, and Anna York for critical and insightful discussions on the initial survey design; and our many data users and collaborators who suggested survey revisions and topics. This material is based on work supported by gifts from Facebook, Google.org, and grants from the Centers for Disease Control and Prevention.

11. Agency for Toxic Substances and Disease Registry, CDC/ATSDR Social Vulnerability Index 2021.https://www.atsdr.cdc.gov/placeandhealth/svi/index.html. Accessed 16 June 2021.

12. A. Reinhart et al., An open repository of real-time COVID-19 indicators. Proc. Natl. Acad. Sci. U.S.A. 118, e2111452118 (2021).

13. P. F. Rebeiro, D. M. Aronoff, M. K. Smith, The impact of state mask-wearing requirements on the growth of COVID-19 cases in the United States. Clin. Infect. Dis. 73 1703-1706 (2021).

14. B. Rader et al., Mask-wearing and control of SARS-CoV-2 transmission in the USA: A cross-sectional study. Lancet Digit. Health 3, e148-e157 (2021).

15. A. Bilinski, E. Emanuel, J. A. Salomon, A. Venkataramani, Better late than never: Trends in COVID-19 infection rates, risk perceptions, and behavioral responses in the USA. J. Gen. Intern. Med. 36, 1825-1828 (2021)

16. A. Flaxman, D. Henning, H. Duber, The relative incidence of COVID-19 in healthcare workers versus non-healthcare workers: Evidence from a web-based survey of Facebook users in the United States. Gates Open Res. 4, 174 (2020).

17. J. Lessler et al., Household COVID-19 risk and in-person schooling. Science $\mathbf{3 7 2}$, 1092-1097 (2021).

18. A. Rodríguez et al., DeepCOVID: An operational deep learning-driven framework for explainable real-time COVID-19 forecasting. Proc. Conf. AAAl Artif. Intell. 35 15393-15400 (2021).

19. D. J. McDonald et al., Can auxiliary indicators improve COVID-19 forecasting and hotspot prediction? Proc. Natl. Acad. Sci. U.S.A. 118, e2111453118 (2021)

20. X. Meng, Statistical paradises and paradoxes in big data (I): Law of large populations, big data paradox, and the 2016 US presidential election. Ann. Appl. Stat. 12, 685-726 (2018). 